\title{
Nucleotide Biosynthesis Process
}

National Cancer Institute

\section{Source}

National Cancer Institute. Nucleotide Biosynthesis Process. NCI Thesaurus. Code C41545.

Nucleotide Biosynthesis consists of activities of biologic molecules or complexes involved in the enzymatic formation of nucleoside phosphates, the monomeric building blocks from which DNA or RNA polymers are constructed. 